\title{
Latest physics design of the HEPS accelerator
}

\author{
Yi Jiao ${ }^{1,2}$
}

Published online: 14 October 2020

(c) Institute of High Energy Physics, Chinese Academy of Sciences 2020

This issue of Journal of Radiation Detection Technology and Methods includes four articles on the physics design of the HEPS accelerator; they are: Modification and optimization of the storage ring lattice of the High Energy Photon Source, Design of the HEPS booster lattice, Physics design of HEPS LINAC and The Transfer Line Design for the HEPS Project.

Accelerator-based light sources had been the most widely used and successful X-ray sources in the past twentieth century. Nowadays, the fourth-generation storage ring light source is the development frontier of international synchrotron radiation light source field. By adopting compact multi-bend achromats (MBAs), it is feasible to reduce the natural emittance of electron beam by 1-2 orders of magnitude compared with the existing third-generation light sources, approaching the diffraction limit of X-rays. Thus, this new-generation light source is also called diffractionlimited storage ring (DLSR) light source. The DLSR light source has the potential and capability to provide X-rays with unprecedented high brightness and coherence for multidisciplinary frontier research and national strategic needs.

Especially since 2009, when the world's first MBA light source, the MAX IV in Sweden broke through, DLSR has attracted worldwide interests and inspired great efforts to propose and construct MBA light sources. For the High Energy Photon Source (HEPS), the first DLSR light source in China, after more or less one decade from its proposal in 2008, the preliminary design report (PDR) was released in 2018 and the facility construction started in mid-2019. The HEPS is a $6 \mathrm{GeV}$, green field light source, with the aim of generating X-ray synchrotron radiations with brightness of higher than $1 \times 10^{22} \mathrm{ph} /\left(\mathrm{s} \mathrm{mm}^{2} \mathrm{mrad}^{2} 0.1 \% \mathrm{BW}\right)$ and photon energy of up to $300 \mathrm{keV}$ at the designed beam current of $200 \mathrm{~mA}$. The HEPS accelerator is comprised of a storage ring with a circumference of $\sim 1.3 \mathrm{~km}$ and natural emittance of below

Yi Jiao

jiaoyi@ihep.ac.cn

1 Key Laboratory of Particle Acceleration Physics and Technology, Institute of High Energy Physics, Chinese Academy of Sciences, Beijing, China

2 University of Chinese Academy of Sciences, Beijing, China
100 pm.rad, a full energy booster, a 500-MeV Linac and three transfer lines. And 14 beamlines will be built in the current Phase I stage.

During the hardware and engineering design of the HEPS, a series of engineering and technical problems emerge. To deal with these challenges, the physics design of HEPS was updated on the basis of that in the PDR. This special topic focuses on the latest designs of the storage ring, booster, Linac and transfer lines, consisting of four research articles. First, Jiao et al. introduce the background, reasons and results of modifications of the HEPS storage ring lattice based on the PRD design. Second, Peng et al. introduce the main considerations and evolution of the HEPS booster lattice design and report the efforts of further pushing down the natural emittance in the booster. Third, Meng et al. summarize the evolution and especially the latest version of the HEPS Linac design. And the fourth, Guo et al. introduce the latest design of transfer lines connecting the Linac, booster and storage ring.

Up to date, a variety of DLSR light sources have been proposed at home and aboard. We hope that this issue can provide a comprehensive insight to the latest design of the HEPS, which would be useful to scientists of related field. Finally, we would like to thank all the authors, reviewers, editors and publishing staff for their great efforts for the publication of the four articles. 